\title{
Design, Synthesis, and Evaluation of a Biomimetic Artificial
}

\section{Photolyase Model}

\author{
Olaf Wiest,${ }^{\$, *}$ Christopher B. Harrison, ${ }^{\$}$ Nicolas J. Saettel,${ }^{\$}$ Radek Cibulka, ${ }^{\#}$ Mirjam Sax ${ }^{\#}$ and \\ Burkhard König ${ }^{\#, *}$
}

Department of Chemistry and Biochemistry, University of Notre Dame, Notre Dame, Indiana 465565670 (USA) and Institut für Organische Chemie, Universität Regensburg, Universitätsstr. 31, D-93040 Regensburg (Germany)

owiest@nd.edu,burkhard.koenig@chemie.uni-regensburg.de

Synthesis and characterization of $\mathbf{5 a , 6 , 7 , 8}$

S2

Synthesis and characterization of $\mathbf{2 a}, \mathbf{9}$

Synthesis and characterization of $\mathbf{5 b}, \mathbf{1 0 , 1 1}$

S4

Synthesis and characterization of $\mathbf{2 b}, \mathbf{1 2 , 1 3}$ S5

IR Spectrum of $\mathbf{2 a}$, UV Spectra of $\mathbf{2 a}$ and $\mathbf{2 b}$ S6

Binding Studies of $\mathbf{2 a}$ S7

Binding Studies of $\mathbf{2 b}$ 


\section{Synthesis of 2a:}

Butyl-(4,5-dimethyl-2-nitro-phenyl)-amine (6)

$n$-Butylamine $(2.8 \mathrm{ml}, 28.3 \mathrm{mmol})$ are added to a solution of $3.5 \mathrm{~g}$ (17.8 mmol) of 4,5-dimethyl-1,2dinitrobenzene $^{1}$ in $440 \mathrm{ml}$ pyridine and refluxed for $72 \mathrm{~h}$. The dark yellow solution is diluted with $500 \mathrm{ml}$ of chloroform and washed five times with $10 \%$ aqueous citric acid. The organic layer is dried over magnesium sulfate and the solvent is evaporated. Chromatographic purification on silica (ethyl acetate/ petrol ether 95:5) yields $2.31 \mathrm{~g}(10.4 \mathrm{mmol}, 59 \%)$ of $\mathbf{6}$ as a light red solid. mp. $55-57^{\circ} \mathrm{C}$; IR $(\mathrm{KBr}) \widetilde{v}=3371 \mathrm{~cm}^{-1}, 2958,2926$, 2862, 1628, 1570, 1503, 1332, 860; UV/Vis $\left(\mathrm{CH}_{3} \mathrm{CN}\right): \lambda_{\max }(\lg \varepsilon)=236$ (4.324), 292 (3.848), 444 (3.789); ${ }^{1} \mathrm{H}-$ $\operatorname{NMR}(250 \mathrm{MHz}): \delta=1.01\left(\mathrm{t},{ }^{3} J=7.51 \mathrm{~Hz}, 3 \mathrm{H}\right), 1.50(\mathrm{~m}, 2 \mathrm{H}), 1.70(\mathrm{~m}, 2 \mathrm{H}), 2.17(\mathrm{~s}, 3 \mathrm{H}), 2.27(\mathrm{~s}, 3 \mathrm{H}), 3.28$ $\left(\mathrm{t},{ }^{3} \mathrm{~J}=6.98 \mathrm{~Hz}, 2 \mathrm{H}\right), 6.62(\mathrm{~s}, 1 \mathrm{H}), 7.92(\mathrm{~s}, 1 \mathrm{H}), 7.97(\mathrm{~m}, 1 \mathrm{H}) ;{ }^{13} \mathrm{C}-\mathrm{NMR}(63 \mathrm{MHz}): \delta=13.8(+), 18.6(+)$, $20.3(-), 20.8(+), 31.1(-), 42.8(-), 114.1(+), 126.5(+), 124.2\left(\mathrm{C}_{\text {quat }}\right), 129.7\left(\mathrm{C}_{\text {quat }}\right), 144.4\left(\mathrm{C}_{\text {quat }}\right), 147.2\left(\mathrm{C}_{\text {quat }}\right)$; MS (EI): m/z (\%): $222(32)\left[\mathrm{M}^{+\bullet}\right], 179(100)\left[\mathrm{M}^{+}-43\left(\mathrm{C}_{3} \mathrm{H}_{7}\right)\right], 176(3)\left[\mathrm{M}^{+}-46\left(\mathrm{NO}_{2}\right)\right] ; \mathrm{C}_{12} \mathrm{H}_{18} \mathrm{~N}_{2} \mathrm{O}_{2}(222.29)$ : calc. C 64.82, H 8.17, N 12.61; exp. C 64.76, H 8.13, N 12.43 .

\section{0-Butyl-7,8-dimethyl-10H-benzo[g]pteridin-2,4-dione (5a)}

Butyl-(4,5-dimethyl-2-nitro-phenyl)-amine $(\mathbf{6})(800 \mathrm{mg}, 3.6 \mathrm{mmol})$ is dissolved in acetic acid and hydrogenated in the presence of $100 \mathrm{mg}$ palladium on carbon for $24 \mathrm{~h}$ at room temperature with a hydrogen pressure of $4 \mathrm{bar}$. The air sensitive product of the reaction, butyl-4,5-dimethyl-benzene-1,2-diamine, is immediately filtered through celite and mixed with $2 \mathrm{~g}(32.3 \mathrm{mmol})$ of $\mathrm{H}_{3} \mathrm{BO}_{3}$ and $1.52 \mathrm{~g}(9.5 \mathrm{mmol})$ alloxane monohydrate. After stirring at room temperature for $24 \mathrm{~h}$, the reaction mixture is diluted with $220 \mathrm{ml}$ of $\mathrm{CHCl}_{3}$ and washed three times with water. After drying of the organic layer over $\mathrm{MgSO}_{4}$, the solvent is evaporated and $\mathbf{5}$ is isolated as an orange powder through recrystallization from acetic acid/water $30: 70$ to yield $587 \mathrm{mg}(2.0 \mathrm{mmol}, 56 \%) \mathrm{mp}$. $>240^{\circ} \mathrm{C}$; IR $(\mathrm{KBr}) \tilde{v}=3170 \mathrm{~cm}^{-1}, 2966,2876,2802,1703,1681,1576,1504,1342,846$; UV/Vis $\left(\mathrm{CH}_{3} \mathrm{CN}\right)$ : $\lambda_{\max }(\lg \varepsilon)=224 \mathrm{~nm}$ (4.405), 268 (4.393), 342 (3.790), 442 (3.967); ${ }^{1} \mathrm{H}-\mathrm{NMR}$ (250 MHz, [D $]$ ]-DMSO): $\delta=0.96$ $\left(\mathrm{t},{ }^{3} J=7.30 \mathrm{~Hz}, 3 \mathrm{H}\right), 1.49(\mathrm{~m}, 2 \mathrm{H}), 1.70(\mathrm{~m}, 2 \mathrm{H}), 2.40(\mathrm{~s}, 3 \mathrm{H}), 2.51(\mathrm{~s}, 3 \mathrm{H}), 4.57\left(\mathrm{t},{ }^{3} J=7.85 \mathrm{~Hz}, 2 \mathrm{H}\right), 7.78$ $(\mathrm{s}, 1 \mathrm{H}), 7.87(\mathrm{~s}, 1 \mathrm{H}), 11.30(\mathrm{~s}, 1 \mathrm{H}) ;{ }^{13} \mathrm{C}-\mathrm{NMR}\left(63 \mathrm{MHz},\left[\mathrm{D}_{6}\right]-\mathrm{DMSO}\right): \delta=13.6(+), 18.7(+), 19.4(-), 20.5$ $(+), 28.5(-), 43.9(-), 115.9(+), 130.6\left(\mathrm{C}_{\text {quat }}\right), 130.6(+), 133.6\left(\mathrm{C}_{\text {quat }}\right), 135.6\left(\mathrm{C}_{\text {quat }}\right), 136.9\left(\mathrm{C}_{\text {quat }}\right), 146.5\left(\mathrm{C}_{\text {quat }}\right)$, $149.9\left(\mathrm{C}_{\text {quat }}\right), 155.5\left(\mathrm{C}_{\text {quat }}\right), 159.8\left(\mathrm{C}_{\text {quar }}\right) ; \mathrm{MS}(\mathrm{EI}): \mathrm{m} / \mathrm{z}(\%): 298(15)\left[\mathrm{M}^{+\bullet}\right], 256(46)\left[\mathrm{M}^{+}-42\left(\mathrm{C}_{3} \mathrm{H}_{6}\right)\right], 242(100)$ $\left[\mathrm{M}^{+}-56\left(\mathrm{C}_{4} \mathrm{H}_{8}\right)\right] ; \mathrm{C}_{16} \mathrm{H}_{18} \mathrm{~N}_{4} \mathrm{O}_{2}$ (298.34): calc. C 64.40, H 6.08, N 18.79; exp. C 63.91, H 6.08, N 18.42;

\section{0-Bromoacetyl-1,4,7,10-tetraazacyclododecan-1,4,7-tricarboxylic acid-tri-tert-butylester $(7)^{2}$}

1,4,7,10-Tetraazacyclododecan-1,4,7-tricarboxylic acid tri-tert-butylester 4 (1 g, $2.12 \mathrm{mmol}){ }^{3}$ bromo acetic acid (295 mg, $2.12 \mathrm{mmol}$ ) and DCC (437 mg, $2.12 \mathrm{mmol}$ ) were stirred in $25 \mathrm{ml}$ of $\mathrm{CH}_{2} \mathrm{Cl}_{2}$ at room temperature for $15 \mathrm{~h}$ under a nitrogen atmosphere. The precipitate is filtered off and the filtrate is washed with $2 \mathrm{M} \mathrm{NaOH}$ solution. The organic layer is dried over $\mathrm{Na}_{2} \mathrm{SO}_{4}$, the solvent is evaporated and $\mathbf{7}$ is isolated as a white solid by chromatography on silica (ethyl acetate/ petrol ether 3:1). Yield: $893 \mathrm{mg}(1.5 \mathrm{mmol}, 70 \%), \mathrm{mp} .55^{\circ} \mathrm{C} .{ }^{1} \mathrm{H}-\mathrm{NMR}$ $(250 \mathrm{MHz}): \delta=1.46(\mathrm{~s}, 18 \mathrm{H}) ; 1.49(\mathrm{~s}, 9 \mathrm{H}), 3.38-3.59$ (m, $16 \mathrm{H}), 3.85$ (s, $2 \mathrm{H})$.

\section{0-[2-(10-Butyl-7,8-dimethyl-2,4-dioxo-4,10-dihydro-2H-benzo[g]pteridin-3-yl)-acetyl]-1,4,7,10-tetraaza- cyclododecan-1,4,7- tricarboxylic acid-tri-tert-butylester $(8)$}

A mixture of 5 (384 mg, $1.3 \mathrm{mmol}), 7(765 \mathrm{mg}, 1.3 \mathrm{mmol})$ and $3.25 \mathrm{~g} \mathrm{~K}_{2} \mathrm{CO}_{3}$ in $50 \mathrm{ml}$ of acetonitrile were stirred under a nitrogen atmosphere at room temperature for 6 days. After filtration of the $\mathrm{K}_{2} \mathrm{CO}_{3}$, the solvent is evaporated and $\mathbf{8}$ is isolated as a dark orange solid by chromatography on silica (ethyl acetate/ methanol 10:1). Yield: $616 \mathrm{mg}(0.76 \mathrm{mmol}, 58 \%)$. mp. $180^{\circ} \mathrm{C}$; IR $(\mathrm{KBr}) \tilde{v}=2973 \mathrm{~cm}^{-1}, 2931,1667,1584,1251,855$; UV/Vis

1 a) Carell, T.; Schmid, H.; Reinhard, M. J. Org. Chem. 1998, 63, 8741. b) Case, F. J. Am. Chem. Soc. 1948, 70, 3994. c) Bamberger, E.; Hübner, R. Ber. Dtsch. Chem. Ges. 1903, 36, 3803. d) Kuhn, R.; v. Klaveren, W. Ber. Dtsch. Chem. Ges. 1938, 71, 779.

2 König, B.; Pelka, M.; Rödel, M.; Klein, M;. Dix, I.; Jones, P. G. J. Incl. Phen. 2000, 37, 39.

3 Kimura, E.; Aoki, S.; Koike, T.; Shiro, M. J. Am. Chem. Soc. 1997, 119, 3068 
$\left(\mathrm{CH}_{3} \mathrm{CN}\right): \lambda_{\max }(\lg \varepsilon)=224 \mathrm{~nm}(4.444), 272$ (4.431), 344 (3.823), 446 (3.960); ${ }^{1} \mathrm{H}-\mathrm{NMR}(250 \mathrm{MHz}): \delta=1.60(\mathrm{t}$, $\left.{ }^{3} J=7.38 \mathrm{~Hz}, 3 \mathrm{H}\right), 1.48(\mathrm{~s}, 27 \mathrm{H}), 1.68(\mathrm{~m}, 2 \mathrm{H}), 1.84(\mathrm{~m}, 2 \mathrm{H}), 1.99(\mathrm{~s}, 3 \mathrm{H}), 2.02(\mathrm{~s}, 3 \mathrm{H}), 3.44-3.68$ $(\mathrm{m}, 16 \mathrm{H}), 4.68\left(\mathrm{t},{ }^{3} \mathrm{~J}=7.53 \mathrm{~Hz}, 2 \mathrm{H}\right), 4.90(\mathrm{~s}, 2 \mathrm{H}), 7.37(\mathrm{~s}, 1 \mathrm{H}), 8.07(\mathrm{~s}, 1 \mathrm{H}) ;{ }^{13} \mathrm{C}-\mathrm{NMR}(100 \mathrm{MHz}): \delta=13.8$ (+), $14.2(+), 19.5(+), 20.2(-), 21.7(+), 28.5(+), 28.5(+), 29.1(-), 43.5(+), 44.7(-), 50.0-50.4(-), 80.2(+)$, $80.5(+), 115.2(+), 131.0\left(\mathrm{C}_{\text {quat }}\right), 132.9(+), 135.0\left(\mathrm{C}_{\text {quat }}\right), 135.9\left(\mathrm{C}_{\text {quat }}\right), 136.5\left(\mathrm{C}_{\text {quat }}\right), 147.6\left(\mathrm{C}_{\text {quat }}\right), 148.9\left(\mathrm{C}_{\text {quat }}\right)$, $155.4\left(\mathrm{C}_{\text {quat }}\right), 155.6\left(\mathrm{C}_{\text {quat }}\right), 157.7\left(\mathrm{C}_{\text {quat }}\right), 159.8\left(\mathrm{C}_{\text {quat }}\right), 168.8\left(\mathrm{C}_{\text {quat }}\right) ; \mathrm{MS}(\mathrm{ESI}): \mathrm{m} / \mathrm{z}(\%): 217$ (100), 833 (74) $[\mathrm{M}+\mathrm{Na}]^{+}, 811(5)[\mathrm{MH}]^{+}$;

\section{0-Butyl-7,8-dimethyl-3-[2-oxo-2-(1,4,7,10-tetraaza-cyclododec-1-yl)-ethyl]-10H-benzo[g]pteridin-2,4- dione (9)}

Compound 8 is stirred in $15 \mathrm{ml}$ of $\mathrm{CF}_{3} \mathrm{COOH} / \mathrm{CH}_{2} \mathrm{Cl}_{2}$ (1:1) under a nitrogen atmosphere at room temperature for $3 \mathrm{~h}$. The solution is then adjusted to $\mathrm{pH} 12$ using $2 \mathrm{M} \mathrm{NaOH}$ and the aqueous layer is extracted with $\mathrm{CH}_{2} \mathrm{Cl}_{2}$. The organic layer is dried over $\mathrm{Na}_{2} \mathrm{SO}_{4}$, the solvent is evaporated and $\mathbf{9}$ is isolated as a brown-orange solid, yield: $360 \mathrm{mg}(0.71 \mathrm{mmol}, 94 \%) \mathrm{mp} .150^{\circ} \mathrm{C}$; IR $(\mathrm{KBr}) \tilde{v}=3435 \mathrm{~cm}^{-1}, 2927,2855,1707,1655,1582,1546$; UV/Vis $\left(\mathrm{CH}_{3} \mathrm{CN}\right): \lambda_{\max }(\lg \varepsilon)=234 \mathrm{~nm}$ (4.499), $282(4.515), 355$ (3.881), $456(4.037) ;{ }^{1} \mathrm{H}-\mathrm{NMR}(250 \mathrm{MHz}): \delta=1.03(\mathrm{t}$, $\left.{ }^{3} J=7.28 \mathrm{~Hz}, 3 \mathrm{H}\right), 1.56(\mathrm{~m}, 2 \mathrm{H}), 1.85(\mathrm{~m}, 2 \mathrm{H}), 2.44(\mathrm{~s}, 3 \mathrm{H}), 2.56(\mathrm{~s}, 3 \mathrm{H}), 2.75-3.07(\mathrm{~m}, 12 \mathrm{H}), 3.74(\mathrm{~m}, 4$ $\mathrm{H}), 4.69\left(\mathrm{t},{ }^{3} \mathrm{~J}=7.63 \mathrm{~Hz}, 2 \mathrm{H}\right), 7.39(\mathrm{~s}, 1 \mathrm{H}), 8.06(\mathrm{~s}, 1 \mathrm{H}) ;{ }^{13} \mathrm{C}-\mathrm{NMR}(100 \mathrm{MHz}): \delta=13.7(+), 19.5(+), 20.1(-)$, $21.6(+), 29.1(-), 43.1(-), 44.8(-), 45.1-50.9(-), 115.2(+), 131.0\left(\mathrm{C}_{\text {quat }}\right), 132.7(+), 135.1\left(\mathrm{C}_{\text {quat }}\right), 135.4\left(\mathrm{C}_{\text {quat }}\right)$, $136.8\left(\mathrm{C}_{\text {quat }}\right), 148.1\left(\mathrm{C}_{\text {quat }}\right), 148.8\left(\mathrm{C}_{\text {quat }}\right), 155.4\left(\mathrm{C}_{\text {quat }}\right), 159.9\left(\mathrm{C}_{\text {quat }}\right), 168.0\left(\mathrm{C}_{\text {quat }}\right) ; \mathrm{MS}(\mathrm{ESI}): \mathrm{m} / \mathrm{z}(\%): 511(100)$ $[\mathrm{MH}]^{+}, 256(54)[\mathrm{M}+2 \mathrm{H}]^{2+}$;

\section{0-Butyl-7,8-dimethyl-3-[2-oxo-2-(1,4,7,10-tetraaza-cyclododec-1-yl)-ethyl]-10H-benzo[g]pteridin-2,4- dione-zinc (II) bis-perchlorate (2a) ${ }^{4}$}

A solution of $9(109 \mathrm{mg}, 0.21 \mathrm{mmol})$ in $2.5 \mathrm{ml}$ of $\mathrm{MeOH}$ is mixed with a solution of $\mathrm{Zn}\left(\mathrm{ClO}_{4}\right)_{2} \bullet 6 \mathrm{H}_{2} \mathrm{O}$ $(78.2 \mathrm{mg}, 0.21 \mathrm{mmol})$ in $1.5 \mathrm{ml}$ of $\mathrm{MeOH}$ and refluxed for $1.5 \mathrm{~h}$ under nitrogen. The solvent is evaporated almost to dryness and $\mathbf{1}$ is isolated as an orange solid by filtration and drying in vacuum. Yield: $150 \mathrm{mg}$ $(0.19 \mathrm{mmol}, 92 \%), \mathrm{mp} .160^{\circ} \mathrm{C}$; IR $(\mathrm{KBr}) \tilde{v}=3553 \mathrm{~cm}^{-1}, 2931,1655,1581,1545,1121 ; \mathrm{UV} / \mathrm{Vis}\left(\mathrm{CH}_{3} \mathrm{CN}\right): \lambda_{\max }$ $(\lg \varepsilon)=234 \mathrm{~nm}$ (4.651), 282 (4.697), 356 (4.076), 456 (4.217); ${ }^{1} \mathrm{H}-\mathrm{NMR}\left(250 \mathrm{MHz}, \mathrm{CD}_{3} \mathrm{CN}\right): \delta=1.01\left(\mathrm{t},{ }^{3} J=\right.$ $7.36 \mathrm{~Hz}, 3 \mathrm{H}), 1.52(\mathrm{~m}, 2 \mathrm{H}), 1.78(\mathrm{~m}, 2 \mathrm{H}), 2.44(\mathrm{~s}, 3 \mathrm{H}), 2.55(\mathrm{~s}, 3 \mathrm{H}), 2.71-4.04(\mathrm{~m}, 16 \mathrm{H}), 4.62\left(\mathrm{t},{ }^{3} J=7.68\right.$ $\mathrm{Hz}, 2 \mathrm{H}), 4.99$ (s, $2 \mathrm{H}), 7.67$ (s, $1 \mathrm{H}), 7.90$ (s, $1 \mathrm{H}) ;{ }^{13} \mathrm{C}-\mathrm{NMR}\left(100 \mathrm{MHz}, \mathrm{CD}_{3} \mathrm{CN}\right): \delta=14.1(+), 19.4(+), 20.8(-$ ), 21.4 (+), $29.9(-), 44.3(-), 44.7(-), 46.1-47.5(-), 117.2(+), 132.4\left(\mathrm{C}_{\text {quat }}\right), 132.5(+), 136.5\left(\mathrm{C}_{\text {quat }}\right), 138.8$ $\left(\mathrm{C}_{\text {quat }}\right), 150.1\left(\mathrm{C}_{\text {quat }}\right), 156.9\left(\mathrm{C}_{\text {quat }}\right), 161.1\left(\mathrm{C}_{\text {quat }}\right), 171.5\left(\mathrm{C}_{\text {quat }}\right), 175.0\left(\mathrm{C}_{\text {quat }}\right) ; \mathrm{MS}(\mathrm{ESI}): \mathrm{m} / \mathrm{z}(\%): 609(100)$ $[\mathrm{MZn}+\mathrm{Cl}]^{+}, 573(26)[\mathrm{MH}+\mathrm{Zn}]^{+} ; 511(60)[\mathrm{M}-\mathrm{Zn}+\mathrm{H}]^{+}, 256(88)[\mathrm{M}+2 \mathrm{H}]^{2+}, 287(51)[\mathrm{M}+\mathrm{Zn}]^{2+}$; HR-MS: calc. $\mathrm{C}_{26} \mathrm{H}_{38} \mathrm{~N}_{8} \mathrm{O}_{3} \mathrm{ClZn} 609.205$; exp. $\mathrm{C}_{26} \mathrm{H}_{38} \mathrm{~N}_{8} \mathrm{O}_{3} \mathrm{ClZn} 609.203\left[\mathrm{M}-\mathrm{H}_{2} \mathrm{O}-2 \mathrm{ClO}_{4}{ }^{2-}\right]^{+}$ 


\section{Synthesis of $2 b$ :}

$N$-[2-(2-Methoxyethoxy)ethyl]-4,5-dimethyl-2-nitroaniline (10): A solution of 1,2-dimethyl-4,5-dinitro benzene $(2.18 \mathrm{~g}, 11.11 \mathrm{mmol})^{1}$ and 2-(2-methoxyethoxy)ethylamine $(2.10 \mathrm{~g}, 17.62 \mathrm{mmol})^{5}$ in pyridine $(270 \mathrm{ml})$ was heated to reflux for 15 hours. An additional amount of 2-(2-methoxyethoxy)ethylamine (1.05 g, $8.81 \mathrm{mmol})$ was added and the reaction mixture was heated to reflux until all starting material was converted to product (TLC analysis, chloroform - methanol $100: 3$ ). The overall reaction time was $\sim 65$ hours. The reaction mixture was cooled and $400 \mathrm{ml}$ of chloroform were added. The solution was extracted with aqueous citric acid $(10 \%, 10 \mathrm{x}$ $250 \mathrm{ml})$ and with water $(2 \times 250 \mathrm{ml})$ and the organic phase was dried over sodium sulfate. After evaporation of the solvent and purification of the crude product by column chromatography (chloroform - methanol $100: 1$ ), compound $10(2.45 \mathrm{~g}, 82.2 \%)$ was obtained as an orange oil. ${ }^{1} \mathrm{H}$ NMR $\left(\mathrm{CDCl}_{3}\right): \delta=2.17\left(\mathrm{~s}, 3 \mathrm{H}, \mathrm{ArCH}_{3}\right), 2.26$ $\left(\mathrm{s}, 3 \mathrm{H}, \mathrm{ArCH}_{3}\right), 3.40\left(\mathrm{~s}, 3 \mathrm{H}, \mathrm{CH}_{3} \mathrm{O}\right), 3.51\left(\mathrm{t},{ }^{3} \mathrm{~J}(\mathrm{H}, \mathrm{H})=5.8 \mathrm{~Hz}, 2 \mathrm{H}, \mathrm{CH}_{2} \mathrm{~N}\right), 3.59\left(\mathrm{~m}, 2 \mathrm{H}, \mathrm{CH}_{3} \mathrm{OCH}_{2}\right), 3.67(\mathrm{~m}$, $\left.2 \mathrm{H}, \mathrm{CH}_{3} \mathrm{OCH}_{2} \mathrm{CH}_{2}\right), 3.77\left(\mathrm{t},{ }^{3} \mathrm{~J}(\mathrm{H}, \mathrm{H})=5.6 \mathrm{~Hz}, 2 \mathrm{H}, \mathrm{OCH}_{2} \mathrm{CH}_{2} \mathrm{~N}\right), 6.63(\mathrm{~s}, 1 \mathrm{H}, \mathrm{ArH}), 7.92(\mathrm{~s}, 1 \mathrm{H}, \mathrm{ArH}) ;{ }^{13} \mathrm{C}$ NMR (CDCl3): $\delta=18.6(+), 20.7(+), 42.7(-), 59.2(+), 69.3(-), 70.6(-), 71.9(-), 114.1(+), 124.5\left(\mathrm{C}_{\text {quat }}\right)$, $126.5(+), 130.0\left(\mathrm{C}_{\text {quat }}\right), 144.1\left(\mathrm{C}_{\text {quat }}\right), 147.2\left(\mathrm{C}_{\text {quat }}\right)$; $\mathrm{MS}(70 \mathrm{eV}, \mathrm{EI}): \mathrm{m} / z(\%): 268(24)[\mathrm{M}]^{+}, 179(100)$ [M$\left.\mathrm{C}_{4} \mathrm{H}_{9} \mathrm{O}_{2}\right]^{+} ; \mathrm{C}_{13} \mathrm{H}_{20} \mathrm{~N}_{2} \mathrm{O}_{4}$ (268.3): calcd. C 58.19, $\mathrm{H}$ 7.51, N 10.44; found: C 57.61, H 7.24, N 10.27.

10-[2-(2-Methoxyethoxy)ethyl]-7,8-dimethyl-10H-benzo[g]pteridin-2,4-dion (5b): Compound 10 (1.30 g, $4.84 \mathrm{mmol})$ was dissolved in acetic acid $(20 \mathrm{ml})$ and, after addition of $\mathrm{Pd} / \mathrm{C}(10 \%)$, stirred for 23 hours under hydrogen atmosphere ( 6 bar). The reaction mixture was filtered through Celite and boric acid (2.7 g, 43.67 $\mathrm{mmol})$ and alloxane monohydrate $(2.48 \mathrm{~g}, 15.49 \mathrm{mmol})$ were added immediately. The mixture was stirred for 9 hours under nitrogen at room temperature. The solution was diluted with chloroform $(250 \mathrm{ml})$ and water $(50 \mathrm{ml})$ and the separated chloroform phase was washed with water $(3 \times 100 \mathrm{ml})$. The organic phase was dried over magnesium sulfate. After evaporation of the solvent and recrystallization of the crude product from water, compound $\mathbf{5 b}(0.86 \mathrm{~g}, 51.5 \%)$ was obtained as orange crystals; m. p. $229{ }^{\circ} \mathrm{C} .{ }^{1} \mathrm{H} \mathrm{NMR}\left(\mathrm{CDCl}_{3}\right): \delta=2.45(\mathrm{~s}, 3$ $\left.\mathrm{H}, \mathrm{ArCH}_{3}\right), 2.54\left(\mathrm{~s}, 3 \mathrm{H}, \mathrm{ArCH}_{3}\right), 3.28\left(\mathrm{~s}, 3 \mathrm{H}, \mathrm{CH}_{3} \mathrm{O}\right), 3.42\left(\mathrm{~m}, 2 \mathrm{H}, \mathrm{CH}_{3} \mathrm{OCH}_{2}\right), 3.60\left(\mathrm{~m}, 2 \mathrm{H}, \mathrm{CH}_{3} \mathrm{OCH}_{2} \mathrm{CH}_{2}\right)$, $4.02\left(\mathrm{t},{ }^{3} J(\mathrm{H}, \mathrm{H})=5.6 \mathrm{~Hz}, 2 \mathrm{H}, \mathrm{OCH}_{2} \mathrm{CH}_{2} \mathrm{~N}\right), 4.93\left(\mathrm{t},{ }^{3} J(\mathrm{H}, \mathrm{H})=5.8 \mathrm{~Hz}, 2 \mathrm{H}, \mathrm{CH}_{2} \mathrm{~N}\right), 7.77(\mathrm{~s}, 1 \mathrm{H}, \mathrm{ArH}), 8.03(\mathrm{~s}$, $1 \mathrm{H}, \mathrm{Ar} H), 8.46(\mathrm{~s}, 1 \mathrm{H}, \mathrm{NH}) .{ }^{13} \mathrm{C} \mathrm{NMR}\left(\mathrm{CDCl}_{3}\right): \delta=19.5(+), 21.4(+), 45.9(-), 59.0(+), 68.0(-), 70.8(-), 71.8$ $(-), 117.0(+), 132.16(+), 132.22\left(\mathrm{C}_{\text {quat }}\right), 135.0\left(\mathrm{C}_{\text {quat }}\right), 135.8\left(\mathrm{C}_{\text {quat }}\right), 137.1\left(\mathrm{C}_{\text {quat }}\right), 148.1\left(\mathrm{C}_{\text {quat }}\right), 150.3\left(\mathrm{C}_{\text {quat }}\right)$, $155.2\left(\mathrm{C}_{\text {quat }}\right), 159.6\left(\mathrm{C}_{\text {quat }}\right)$. MS (70 eV, EI): $\mathrm{m} / z(\%): 344(2)[\mathrm{M}]^{+}, 242(100)\left[\mathrm{M}_{-} \mathrm{C}_{5} \mathrm{H}_{10} \mathrm{O}_{2}\right]^{+}, 171(58)[\mathrm{M}-$ $\left.\mathrm{C}_{7} \mathrm{H}_{11} \mathrm{NO}_{4}\right]^{+}, 156$ (24) [M-C $\left.{ }_{7} \mathrm{H}_{12} \mathrm{~N}_{2} \mathrm{O}_{4}\right]^{+} ; \mathrm{C}_{17} \mathrm{H}_{20} \mathrm{~N}_{4} \mathrm{O}_{4}$ (344.4): calcd: C 59.29, H 5.85, N 16.27; found: C 58.68, H 5.78, N 16.20.

Tri-tert-butyl 10-(2-\{10-[2-(2-methoxyethoxy)ethyl]-7,8-dimethyl-2,4-dioxo-4,10-dihydro-2H-benzo[g] pteridin-3-yl\}acetyl)-1,4,7,10-tetraazacyclododecan-1,4,7-tricarboxylate (11): A mixture of $\mathbf{5 b}(0.34 \mathrm{~g}, 0.99$ mmol), $7(1.73 \mathrm{~g}, 2.91 \mathrm{mmol})$, potassium carbonate $(1.00 \mathrm{~g})$ in dry dimethylformamide $(50 \mathrm{ml})$ was stirred under nitrogen at room temperature until all compound $\mathbf{1 1}$ was converted to product (4 hours). The reaction was monitored by TLC analysis (ethyl acetate - methanol $5: 2)$. The second portion of $\mathbf{5 b}(0.16 \mathrm{~g}, 0.46 \mathrm{mmol})$ was added and the mixture was stirred at room temperature for an additional 8 hours. The reaction mixture was diluted with chloroform $(200 \mathrm{ml})$, washed with water $(4 \times 150 \mathrm{ml})$ and the organic phase was dried over magnesium sulfate. After evaporation of the solvent and purification of crude product by column chromatography (ethyl acetate - methanol $4: 1)$, compound $\mathbf{1 1}(1.10 \mathrm{~g}, 88.4 \%)$ was obtained as a yellow solid; m. p. $137-139{ }^{\circ} \mathrm{C} ;{ }^{1} \mathrm{H}$ NMR $\left(\mathrm{CDCl}_{3}\right): \delta=1.48\left(\mathrm{~m}, 27 \mathrm{H},\left(\mathrm{CH}_{3}\right)_{3} \mathrm{C}, 2.43\left(\mathrm{~s}, 3 \mathrm{H}, \mathrm{ArCH}_{3}\right), 2.53\left(\mathrm{~s}, 3 \mathrm{H}, \mathrm{ArCH}_{3}\right)\right.$, $3.29\left(\mathrm{~s}, 3 \mathrm{H}, \mathrm{CH}_{3} \mathrm{O}\right), 3.30-3.75\left(\mathrm{~m}, 16 \mathrm{H}, \mathrm{CH}_{2} \mathrm{~N}\right), 3.42\left(\mathrm{~m}, 2 \mathrm{H}, \mathrm{CH}_{3} \mathrm{OCH}_{2} \mathrm{CH}_{2}\right), 3.55\left(\mathrm{~m}, 2 \mathrm{H}, \mathrm{CH}_{3} \mathrm{OCH}_{2}\right), 4.00$ $\left(\mathrm{t},{ }^{3} \mathrm{~J}(\mathrm{H}, \mathrm{H})=5.8 \mathrm{~Hz}, 2 \mathrm{H}, \mathrm{OCH}_{2} \mathrm{CH}_{2} \mathrm{~N}\right), 4.85-4.95\left(\mathrm{~m}, 4 \mathrm{H}, \mathrm{CH}_{2} \mathrm{~N}, \mathrm{CH}_{2} \mathrm{Br}\right), 7.75(\mathrm{~s}, 1 \mathrm{H}, \mathrm{ArH}), 8.04(\mathrm{~s}, 1 \mathrm{H}$, $\mathrm{Ar} H) ;{ }^{13} \mathrm{C}$ NMR $\left(\mathrm{CDCl}_{3}\right): \delta=19.5(+), 21.3(+), 28.42(+), 28.46(+), 28.58(+), 45.4(-), 50.03(-), 50.06(-)$, $50.29(-), 51.50(-), 59.0(+), 68.1(-), 70.7(-), 71.7(-), 80.2\left(\mathrm{C}_{\text {quat }}\right), 80.5\left(\mathrm{C}_{\text {quat }}\right), 116.9(+), 132.1\left(\mathrm{C}_{\text {quat }}\right), 132.2$ $(+), 134.9\left(\mathrm{C}_{\text {quat }}\right), 135.5\left(\mathrm{C}_{\text {quat }}\right), 136.6\left(\mathrm{C}_{\text {quat }}\right), 146.7\left(\mathrm{C}_{\text {quat }}\right), 147.5\left(\mathrm{C}_{\text {quat }}\right), 149.0\left(\mathrm{C}_{\text {quat }}\right), 155.2\left(\mathrm{C}_{\text {quat }}\right), 155.6\left(\mathrm{C}_{\text {quat }}\right)$, $159.8\left(\mathrm{C}_{\text {quat }}\right)$; $\mathrm{MS}\left(\mathrm{ESI}, \mathrm{CH}_{2} \mathrm{Cl}_{2}+\mathrm{CH}_{3} \mathrm{OH}+\mathrm{CH}_{3} \mathrm{COONH}_{4}\right): \mathrm{m} / z(\%): 858$ (100) $\left[\mathrm{MH}^{+}\right], 758$ (26) [M-C $\mathrm{H}_{4} \mathrm{H}_{7^{-}}$ $\left.\mathrm{CO}_{2}{ }^{+}\right] ; \mathrm{C}_{42} \mathrm{H}_{64} \mathrm{~N}_{8} \mathrm{O}_{11}$ (857.0): calcd: C 58.86, H 7.53, N 13.07; found: C 57.65, H 7.28, N 12.68.

5 a) Pedersen, C. J. J. Am. Chem. Soc. 1967, 89, 7017. b) Heimann, U.; Herzhoff, M.; Vögtle, F. Chem. Ber. 1979, 112, 1392 
10-[2-(2-Methoxyethoxy)ethyl]-7,8-dimethyl-3-[2-oxo-2-(1,4,7,10-tetraazacyclododec-1-yl)ethyl]- $10 \mathrm{H}$ benzo[g] pteridin-2,4-dione. $3 \mathbf{C F}_{3} \mathbf{C O O H}(12)$ : A solution of $11(0.250 \mathrm{~g}, 0.29 \mathrm{mmol})$ in a mixture of dichloromethane $(5.0 \mathrm{ml})$ and trifluoroacetic acid $(5.0 \mathrm{ml})$ was stirred for 3 hours under nitrogen at room temperature. The solvents were evaporated and the remaining solid was dissolved in water $(10 \mathrm{ml})$ and washed with dichloromethane $(10 \mathrm{ml})$. The water was evaporated under reduced pressure and the remaining solid was dried in vacuo. Product $12(0.26 \mathrm{~g}, 99.1 \%)$ was obtained as yellow solid. M. p. $69-70{ }^{\circ} \mathrm{C} ;{ }^{1} \mathrm{H} \mathrm{NMR}(600 \mathrm{MHz}$, $\left.\mathrm{CD}_{3} \mathrm{CN}\right): \delta=2.43\left(\mathrm{~s}, 3 \mathrm{H}, \mathrm{ArCH}_{3}\right), 2.55\left(\mathrm{~s}, 3 \mathrm{H}, \mathrm{ArCH}_{3}\right), 3.19\left(\mathrm{~s}, 3 \mathrm{H}, \mathrm{CH}_{3} \mathrm{O}\right), 3.22\left(\mathrm{~m}, 8 \mathrm{H}, \mathrm{CH}_{2} \mathrm{~N}\right), 3.26(\mathrm{~m}, 4$ $\left.\mathrm{H}, \mathrm{CH}_{2} \mathrm{~N}\right), 3.37\left(\mathrm{t},{ }^{3} \mathrm{~J}(\mathrm{H}, \mathrm{H})=4.7 \mathrm{~Hz}, 2 \mathrm{H}, \mathrm{CH}_{3} \mathrm{OCH}_{2}\right), 3.56\left(\mathrm{t},{ }^{3} \mathrm{~J}(\mathrm{H}, \mathrm{H})=4.6 \mathrm{~Hz}, 2 \mathrm{H}, \mathrm{CH}_{3} \mathrm{OCH}_{2} \mathrm{CH}_{2}\right), 3.69(\mathrm{~m}, 2$ $\left.\mathrm{H}, \mathrm{CH}_{2} \mathrm{NC}=\mathrm{O}\right), 3.88\left(\mathrm{~m}, 2 \mathrm{H}, \mathrm{CH}_{2} \mathrm{NC}=\mathrm{O}\right), 3.91\left(\mathrm{t},{ }^{3} \mathrm{~J}(\mathrm{H}, \mathrm{H})=5.5 \mathrm{~Hz}, 2 \mathrm{H}, \mathrm{OCH}_{2} \mathrm{CH}_{2} \mathrm{~N}\right), 4.85\left(\mathrm{t},{ }^{3} J(\mathrm{H}, \mathrm{H})=5.5\right.$ $\left.\mathrm{Hz}, 2 \mathrm{H}, \mathrm{CH}_{2} \mathrm{~N}\right), 4.89\left(\mathrm{~s}, 2 \mathrm{H}, \mathrm{NCH}_{2} \mathrm{C}=\mathrm{O}\right), 7.84(\mathrm{~s}, 1 \mathrm{H}, \mathrm{ArH}), 7.89(\mathrm{~s}, 1 \mathrm{H}, \mathrm{ArH}) ;{ }^{13} \mathrm{C} \mathrm{NMR}\left(150 \mathrm{MHz}, \mathrm{CD}_{3} \mathrm{CN}\right)$ : $18.2(+), 20.3(+), 42.5(-), 43.3(-), 44.1(-), 44.3(-), 44.9(-), 45.0(-) 45.8(-), 46.35(-), 46.43(-), 47.5(-), 57.8$ (+), $67.0(-), 70.3(-), 71.3(-), 115.7\left(\mathrm{q}, C \mathrm{~F}_{3}\right), 116.9(+), 131.1(+), 131.9\left(\mathrm{C}_{\text {quat }}\right), 135.07\left(\mathrm{C}_{\text {quat }}\right), 135.10\left(\mathrm{C}_{\text {quat }}\right)$, $137.5\left(\mathrm{C}_{\text {quat }}\right), 148.5\left(\mathrm{C}_{\text {quat }}\right), 149.1\left(\mathrm{C}_{\text {quat }}\right), 155.7\left(\mathrm{C}_{\text {quat }}\right), 160.1\left(\mathrm{C}_{\text {quat }}\right), 160.4\left(\mathrm{q}, \mathrm{CF}_{3} \mathrm{COO}^{-}\right), 169.8\left(\mathrm{C}_{\text {quat }}\right)$; $\mathrm{MS}(\mathrm{ESI}$, $\left.\mathrm{CH}_{3} \mathrm{CN}\right): \mathrm{m} / \mathrm{z}$ (\%): 557 (100) $\left[\mathrm{MH}-3 \mathrm{CF}_{3} \mathrm{COOH}\right]^{+}, 279$ (13) $\left[\mathrm{MH}_{2}-3 \mathrm{CF}_{3} \mathrm{COOH}\right]^{2+}, 186$ (24) $\left[\mathrm{MH}_{3}-\right.$

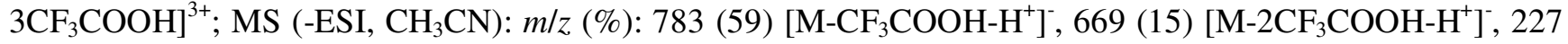
(100) [ $\left.2 \mathrm{CF}_{3} \mathrm{COOH}-\mathrm{H}^{+}\right]^{-} ; \mathrm{C}_{33} \mathrm{H}_{43} \mathrm{~F}_{9} \mathrm{~N}_{8} \mathrm{O}_{11}$ (898.8): calcd: $\mathrm{C} 44.10, \mathrm{H} 4.82, \mathrm{~N} 12.47$; found: $\mathrm{C} 43.96 \mathrm{H} 4.97, \mathrm{~N}$ 12.71 .

\section{0-[2-(2-Methoxyethoxy)ethyl]-7,8-dimethyl-3-[2-oxo-2-(1,4,7,10-tetraazacyclododec-1-yl)ethyl]-10H-}

benzo [ $g$ ]pteridin-2,4-dione (13): The salt $12(0.250 \mathrm{~g}, 0.28 \mathrm{mmol})$ was dissolved in water and transferred to the free base 14 by ion exchange (Merck Ion exchanger III, $\mathrm{OH}^{-}$form). The solvent was evaporated under reduced pressure and the remaining solid was dried under vacuum. Product $13(0.112 \mathrm{~g})$ was used without further purification for the preparation of zinc complex. ${ }^{1} \mathrm{H}$ NMR $\left(\mathrm{CD}_{3} \mathrm{CN}\right): \delta=2.44\left(\mathrm{~s}, 3 \mathrm{H}, \mathrm{ArCH}_{3}\right), 2.53(\mathrm{~s}, 3$ $\left.\mathrm{H}, \mathrm{ArCH}_{3}\right), 2.57\left(\mathrm{~m}, 4 \mathrm{H}, \mathrm{CH}_{2} \mathrm{~N}\right), 2.64\left(\mathrm{~m}, 4 \mathrm{H}, \mathrm{CH}_{2} \mathrm{~N}\right), 2.77\left(\mathrm{~m}, 4 \mathrm{H}, \mathrm{CH}_{2} \mathrm{~N}\right), 3.19\left(\mathrm{~s}, 3 \mathrm{H}, \mathrm{CH}_{3} \mathrm{O}\right), 3.37(\mathrm{~m}, 2 \mathrm{H}$, $\left.\mathrm{CH}_{3} \mathrm{OCH}_{2} \mathrm{CH}_{2}\right), 3.51\left(\mathrm{~m}, 2 \mathrm{H}, \mathrm{CH}_{2} \mathrm{NC}=\mathrm{O}\right), 3.56\left(\mathrm{~m}, 2 \mathrm{H}, \mathrm{CH}_{3} \mathrm{OCH}_{2}\right), 3.59\left(\mathrm{~m}, 2 \mathrm{H}, \mathrm{CH}_{2} \mathrm{NC}=\mathrm{O}\right), 3.91(\mathrm{t}, J=5.8$ $\left.\mathrm{Hz}, 2 \mathrm{H}, \mathrm{OCH}_{2} \mathrm{CH}_{2} \mathrm{~N}\right), 4.83\left(\mathrm{t}, J=5.8 \mathrm{~Hz}, 2 \mathrm{H}, \mathrm{CH}_{2} \mathrm{~N}\right), 4.96\left(\mathrm{~s}, 2 \mathrm{H}, \mathrm{NCH}_{2} \mathrm{C}=\mathrm{O}\right), 7.80(\mathrm{~s}, 1 \mathrm{H}, \mathrm{ArH}), 7.90(\mathrm{~s}, 1$ $\mathrm{H}, \mathrm{ArH}$ ). MS (ESI, $\left.\mathrm{CH}_{3} \mathrm{CN}\right): \mathrm{m} / z$ (\%): 557.3 (100) [ $\left.\mathrm{MH}^{+}\right]$; HR-MS $\left(\mathrm{C}_{27} \mathrm{H}_{41} \mathrm{~N}_{8} \mathrm{O}_{5}\right)$ : calcd. 557.3200;.found $557.3206 \pm 0.0007$.

10-[2-(2-Methoxyethoxy)ethyl]-7,8-dimethyl-3-[2-oxo-2-(1,4,7,10-tetraazacyclododec-1-yl)ethyl]-10Hbenzo[ $g]$ pteridin-2,4-dione zinc(II) perchlorate (2b): A solution of zinc perchlorate hexahydrate in acetonitrile $(2 \mathrm{ml})$ was added into the solution of $13(0.119 \mathrm{~g}, 0.21 \mathrm{mmol})$ in acetonitrile $(3 \mathrm{ml})$ under nitrogen. The mixture was stirred for $30 \mathrm{~min}$. at $60{ }^{\circ} \mathrm{C}$. After evaporation of the solvents, a red solid was obtained, which was dissolved in hot acetone $\left(60{ }^{\circ} \mathrm{C}\right)$. After cooling the solution solid impurities precipitated. The solid was filtered off and, after evaporation of solvents from the filtrate, product $\mathbf{2 b}(0.090 \mathrm{~g}$, yield starting from 12: 39.4 \%) was obtained as a red solid; m. p. $>260{ }^{\circ} \mathrm{C}$, (decomp.). ${ }^{1} \mathrm{H}$ NMR $\left(\mathrm{CD}_{3} \mathrm{CN}\right): \delta=2.45$ (s, $\left.3 \mathrm{H}, \operatorname{ArCH}_{3}\right), 2.55$ (s, $\left.3 \mathrm{H}, \mathrm{ArCH}_{3}\right), 2.72\left(\mathrm{~m}, 2 \mathrm{H}, \mathrm{CH}_{2} \mathrm{~N}\right), 2.85\left(\mathrm{~m}, 2 \mathrm{H}, \mathrm{CH}_{2} \mathrm{~N}\right), 2.95\left(\mathrm{~m}, 2 \mathrm{H}, \mathrm{CH}_{2} \mathrm{~N}\right), 3.00-3.25\left(\mathrm{~m}, 6 \mathrm{H}, \mathrm{CH}_{2} \mathrm{~N}\right)$, $3.15\left(\mathrm{~s}, 3 \mathrm{H}, \mathrm{CH}_{3} \mathrm{O}\right), 3.34\left(\mathrm{~m}, 2 \mathrm{H}, \mathrm{CH}_{3} \mathrm{OCH}_{2} \mathrm{CH}_{2}\right), 3.53\left(\mathrm{~m}, 2 \mathrm{H}, \mathrm{CH}_{3} \mathrm{OCH}_{2}\right), 3.71\left(\mathrm{~m}, 2 \mathrm{H}, \mathrm{CH}_{2} \mathrm{NC}=\mathrm{O}\right), 3.89(\mathrm{t}$, $\left.J=5.8 \mathrm{~Hz}, 2 \mathrm{H}, \mathrm{OCH}_{2} \mathrm{CH}_{2} \mathrm{~N}\right), 3.94\left(\mathrm{~m}, 2 \mathrm{H}, \mathrm{CH}_{2} \mathrm{NC}=\mathrm{O}\right), 4.88\left(\mathrm{t}, J=5.6 \mathrm{~Hz}, 2 \mathrm{H}, \mathrm{CH}_{2} \mathrm{~N}\right), 4.90(\mathrm{~s}, 2 \mathrm{H}$, $\left.\mathrm{NCH}_{2} \mathrm{C}=\mathrm{O}\right), 7.88(\mathrm{~s}, 1 \mathrm{H}, \mathrm{ArH}), 7.94(\mathrm{~s}, 1 \mathrm{H}, \mathrm{ArH}) .{ }^{13} \mathrm{C} \mathrm{NMR}: 18.2(+), 20.2(+), 43.4(-), 43.5(-), 45.2(-), 45.9$ $(-), 46.0(-), 57.7(+), 66.9(-), 70.2(-), 71.2(-), 117.7(+), 131.0(+), 132.3\left(\mathrm{C}_{\text {quat }}\right), 133.0\left(\mathrm{C}_{\text {quat }}\right), 136.1\left(\mathrm{C}_{\text {quat }}\right)$, $139.8\left(\mathrm{C}_{\text {quat }}\right), 150.2\left(\mathrm{C}_{\text {quat }}\right), 150.7\left(\mathrm{C}_{\text {quat }}\right), 157.8\left(\mathrm{C}_{\text {quat }}\right), 161.0\left(\mathrm{C}_{\text {quat }}\right), 173.8\left(\mathrm{C}_{\text {quat }}\right)$.

MS (ESI, $\left.\mathrm{CH}_{3} \mathrm{CN}\right): \mathrm{m} / \mathrm{z}(\%): 310.1(100)\left[\left(\mathrm{M}-2 \mathrm{ClO}_{4}^{-}\right)^{2+}\right], 557.4(50)\left[\mathrm{LH}^{+}\right], 619.3(16)\left[\left(\mathrm{M}-2 \mathrm{ClO}_{4}^{-}-\mathrm{H}^{+}\right)^{+}\right], 679.4$

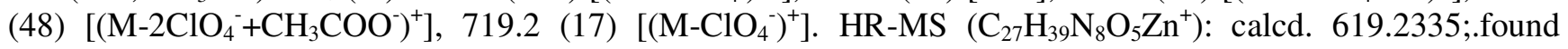
$619.2375 \pm 0.0029$. 
IR-spectrum of $\mathbf{2 a}(\mathrm{KBr})$ :

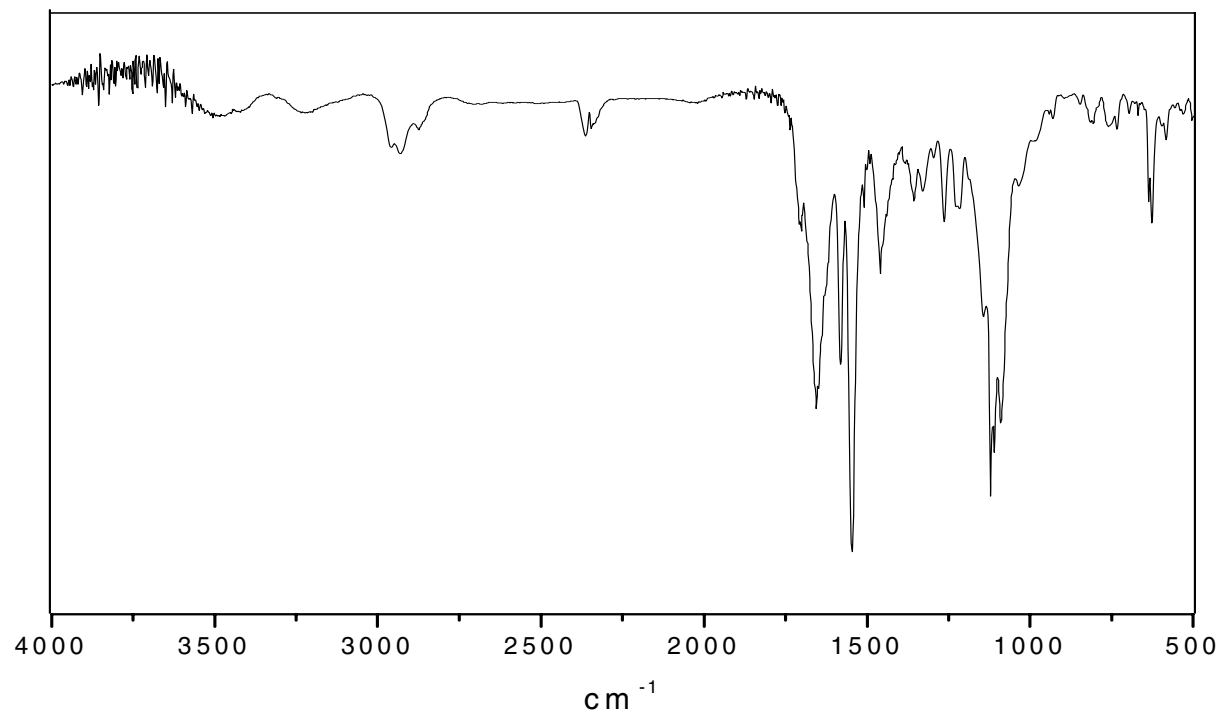

UV-spectrum of $2 \mathbf{a}$ in acetonitrile $\left(\mathrm{c}=5 * 10^{-5} \mathrm{~mol} / \mathrm{l}\right)$

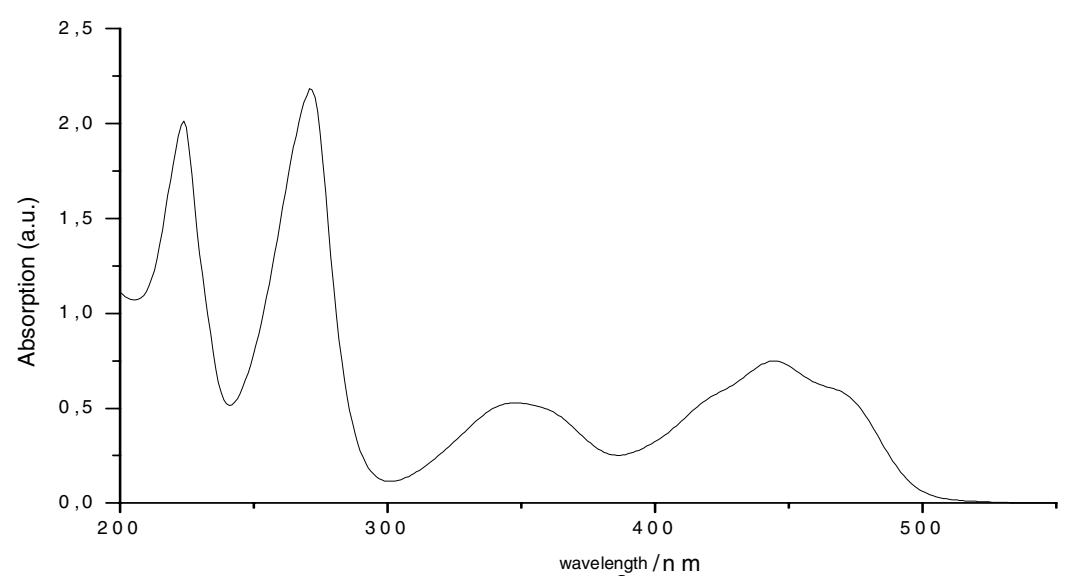

UV spectrum of $\mathbf{2 b}$ in acetonitrile, $c=2.10^{-5} \mathrm{~mol}^{-1}{ }^{-1}$.

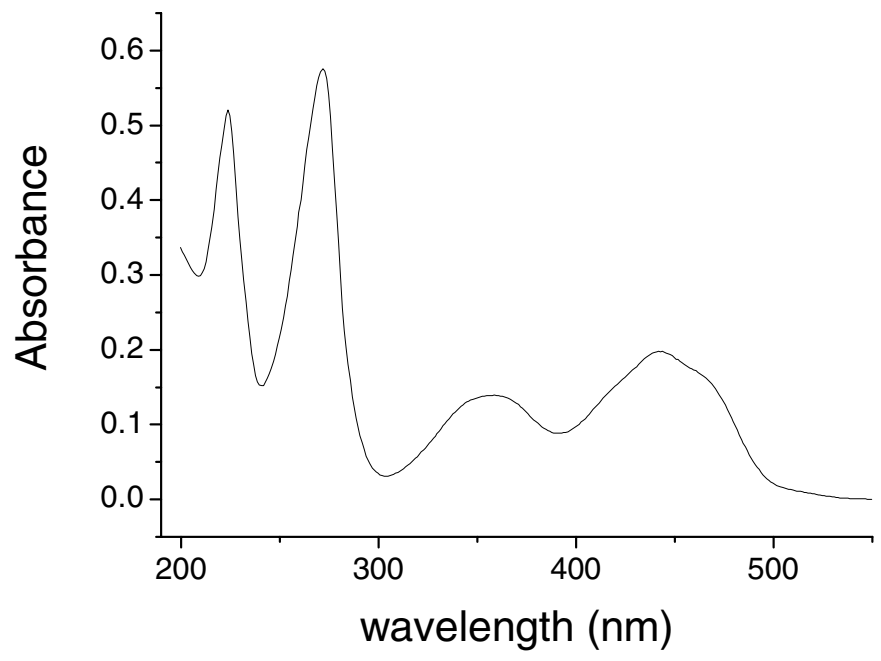


Binding Studies of $\mathbf{2 a}$ :

The binding constants were determined by emission quenching titration measurements using a Varian Cary Eclipse fluorometer. Solutions of 2a and 5a in methanol and acetonitrile were excited at $450 \mathrm{~nm}$. Emissions for 2a were recorded at 515 and $505 \mathrm{~nm}$ and for 5a at $517 \mathrm{~nm}$ and $506 \mathrm{~nm}$ in methanol and acetonitrile, respectively. The results for the titration of $2 \mathrm{a}\left(5 \times 10^{-5} \mathrm{M}^{-1}\right)$ with thymine in methanol are shown below. The stoichiometry is determined via a Job plot, the binding constant is determined from the emission titration by fitting the experimental data to a 1:1 model using standard methods. ${ }^{10}$
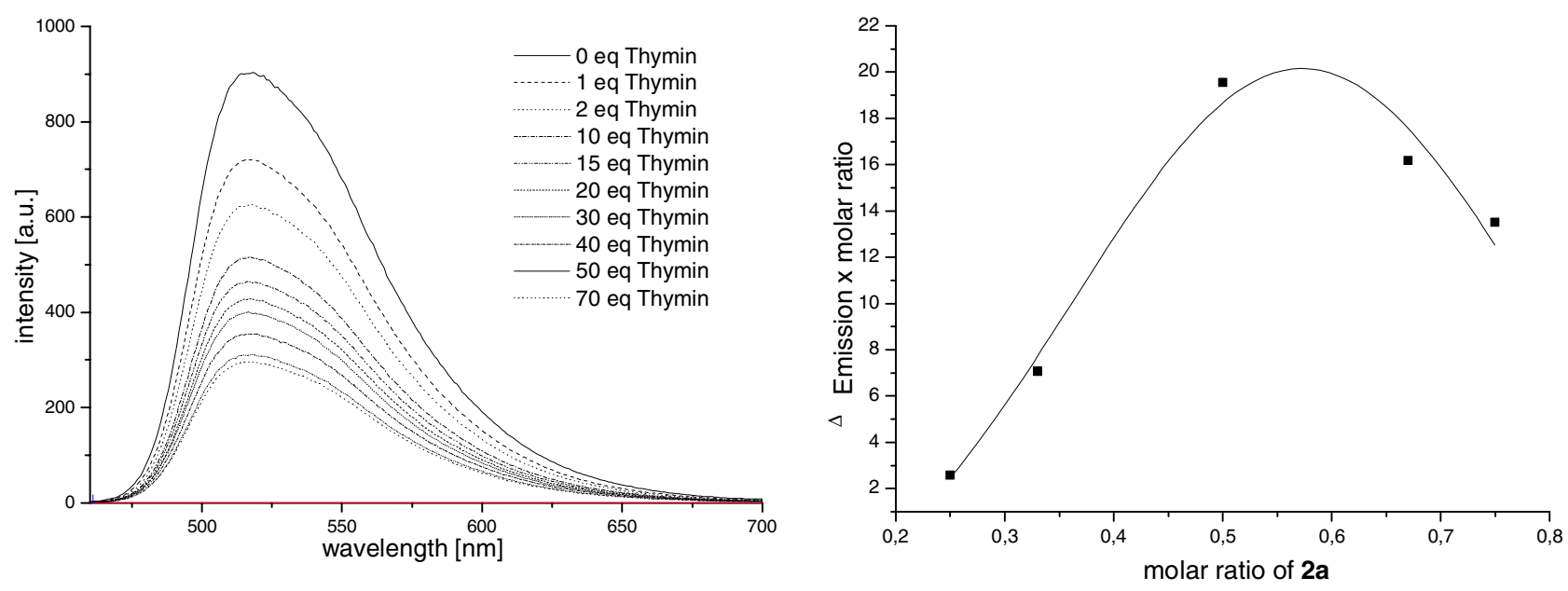

Emission quenching of 2a with thymine (left) and Job's-plot (right)

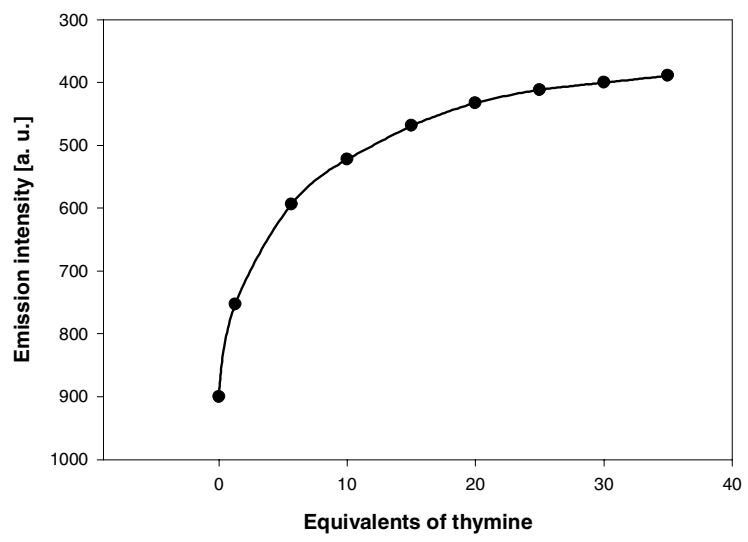

Emission quenching titration of $\mathbf{2 a}$ with thymine

Similarly, the binding constant of $\mathbf{2 a}\left(4 \times 10^{-6} \mathrm{M}^{-1}\right)$ to $\mathbf{3 a}$ in acetonitrile was determined. 

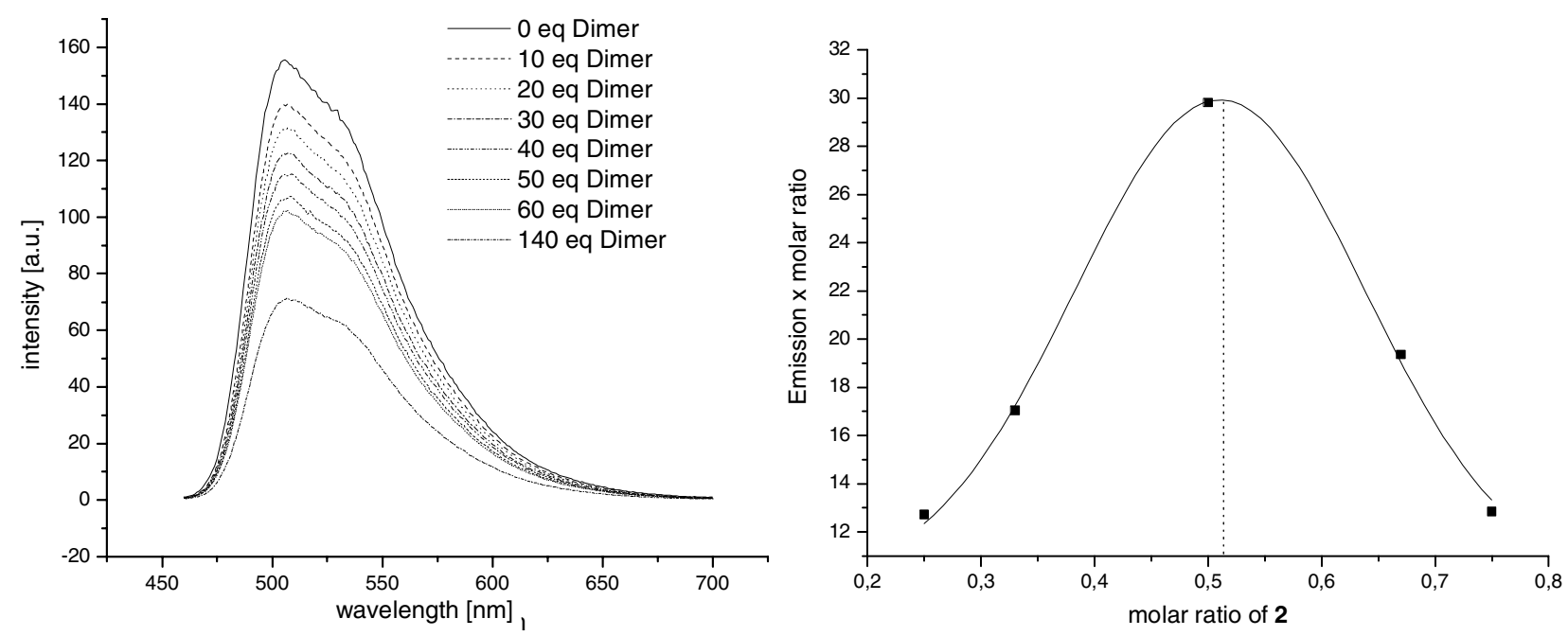

Emission quenching of 2a with 3a (left) and Job's-plot (right)

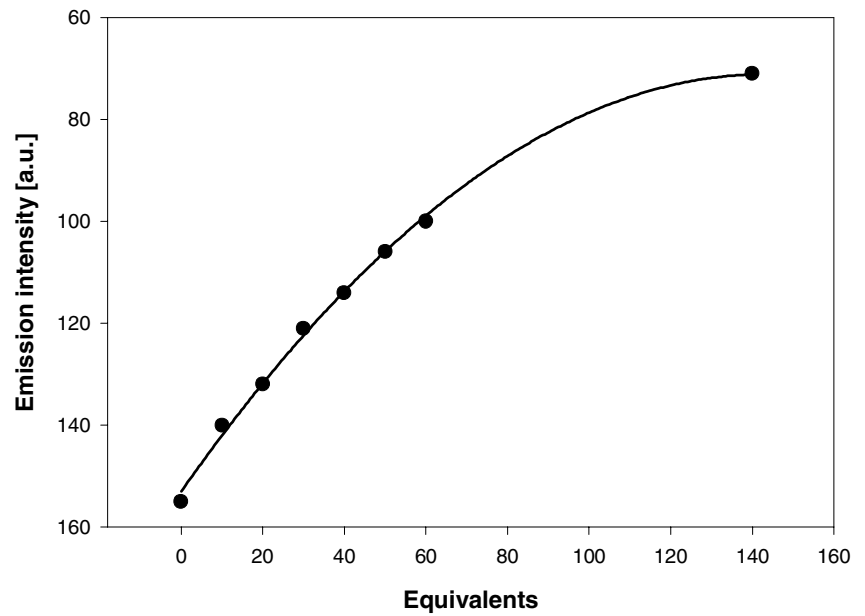

Emission quenching titration of $\mathbf{2 a}$ with $\mathbf{3 a}$ 
Binding Studies for $\mathbf{2 b}$ :

NMR titrations experiments were performed in borate buffer $(\mathrm{c}=0.4$ mol.1-1, $\mathrm{pH}=7.2)$ containing $10 \% \mathrm{D}_{2} \mathrm{O}$ at room temperature. Natrium 2,2,3,3-tetradeutero-3-(trimethylsilyl)propanoate was used as an internal standard. ${ }^{1} \mathrm{H}$ NMR spectra were recorded on Bruker AVANCE 600 at $600 \mathrm{MHz}$ using watergate. The binding constants were calculated from the measured data using the program HypNmr. ${ }^{6}$

Zinc(II) cyclen was used for comparison. Uracil and $\mathbf{3 b}$ show binding equilibria to zinc(II) cyclen with defined stoichiometry. The affinity of uracil is somewhat higher, as expected, if compared to $\mathbf{3 b}$. Compound $\mathbf{2 b}$ shows self-association with a $\lg K=1.8$ as derived from dilution experiments. The binding affinity to uracil was determined to be $\lg K=2.8$. Self association was taken into account. The binding affinity of $\mathbf{2 b}$ to $\mathbf{3 b}$ could not be derived from NMR titration experiments due to substantial signal broadening. As a model for the binding site of $\mathbf{3 b}$ dihydrouracil was used. The titration revealed an affinity to $\mathbf{2 b}$ of $\lg K=2.1$. The affinity is, as expected, smaller than the affinity of uracil.

Table 1. Binding constants of cyclen derivatives with uracil, $\mathbf{3 b}$ and dihydrouracil in borate buffer at pH 7.2.

\begin{tabular}{cccc}
\hline Cyclen derivative & Uracil derivative & $\begin{array}{c}\text { Binding } \\
\text { model }\end{array}$ & $\log \boldsymbol{\beta}_{\mathbf{1}}$ \\
\hline Zinc(II)-cyclen & Uracil & $1: 1$ & $1.95 \pm 0.01$ \\
Zinc(II)-cyclen & $\mathbf{3 b}$ & $1: 1$ & $1.34 \pm 0.08$ \\
2b & Uracil & $1: 1^{a}$ & $2.8 \pm 0.1$ \\
$\mathbf{2 b}$ & Dihydrouracil & $1: 1^{a}$ & $2.1 \pm 0.1$ \\
\hline
\end{tabular}

${ }^{a}$ Self-association of $\mathbf{2 b}(\lg K=1.8)$ is observed in aqueous solution

Fig. 1. Relative chemical shifts in ${ }^{1} \mathrm{H}$ NMR spectra of uracil used for the calculation of binding constants between zinc(II)-cyclen and uracil. Initial concentration $c_{\text {uracil }}=1.147 .10^{-2} \mathrm{~mol}^{-1}{ }^{-1}$.

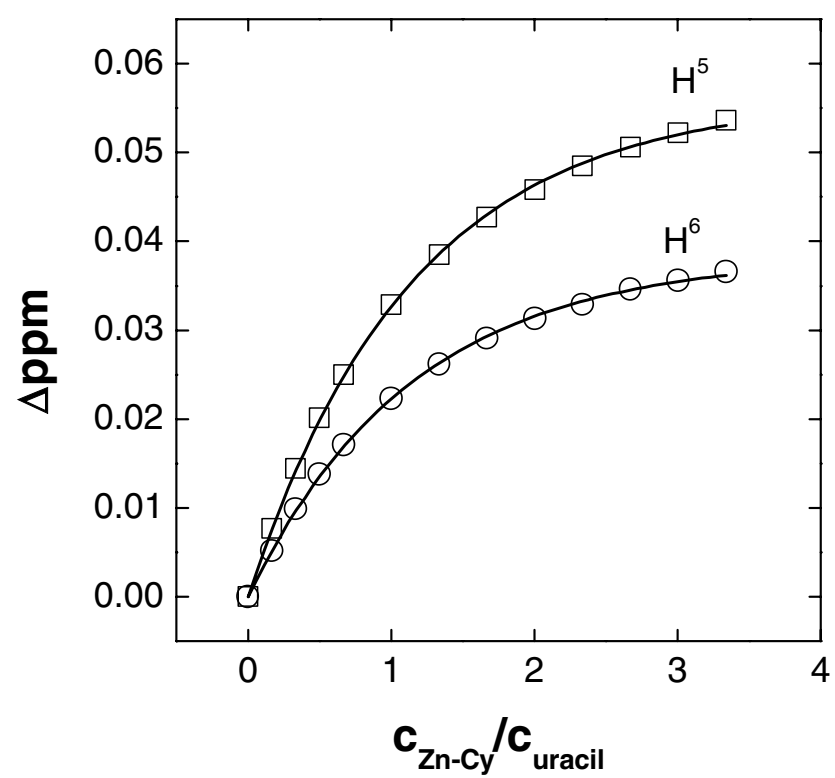

${ }^{6}$ C. Frassineti, S. Ghelli, P. Gans, A. Sabatini, M.S. Moruzzi, A. Vacca, Anal. Biochem. 1995, 231 374-382. 
Fig. 2. Relative chemical shifts in ${ }^{1} \mathrm{H}$ NMR spectra of $\mathbf{3 b}$ used for the calculation of binding constants between zinc(II)-cyclen and $\mathbf{3 b}$. Initial concentration $c_{\text {uracil dimer }}=1.053 .10^{-2} \mathrm{moll}^{-1}$.

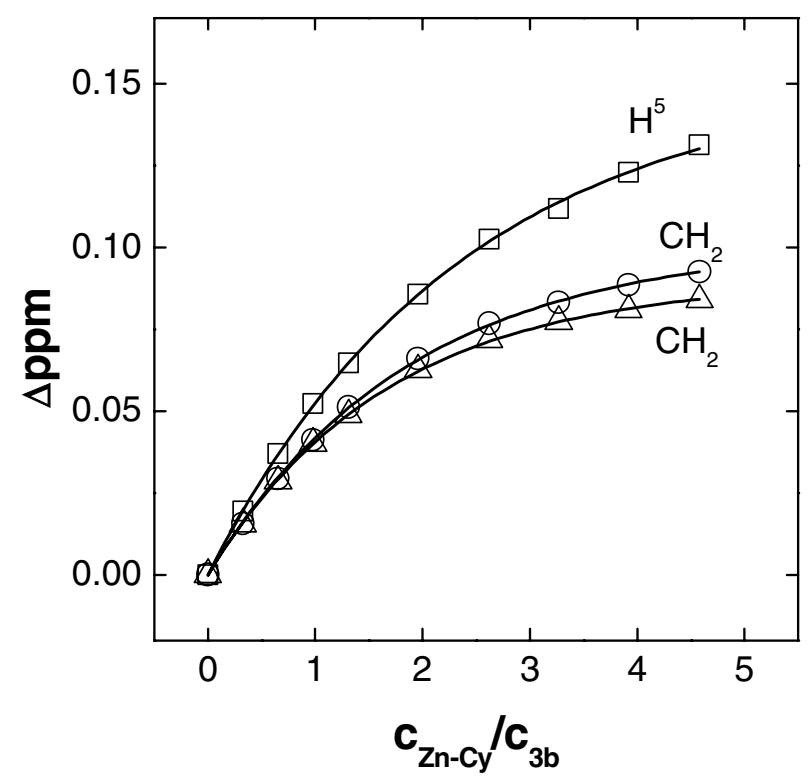

Fig. 3. Relative chemical shifts in ${ }^{1} \mathrm{H}$ NMR spectra of $\mathbf{2} \mathbf{b}$ used for the calculation of binding constants between $\mathbf{2 b}$ and uracil. Initial concentration $c_{2 \mathrm{~b}}=1.050 \cdot 10^{-2} \mathrm{~mol}^{-1}$.

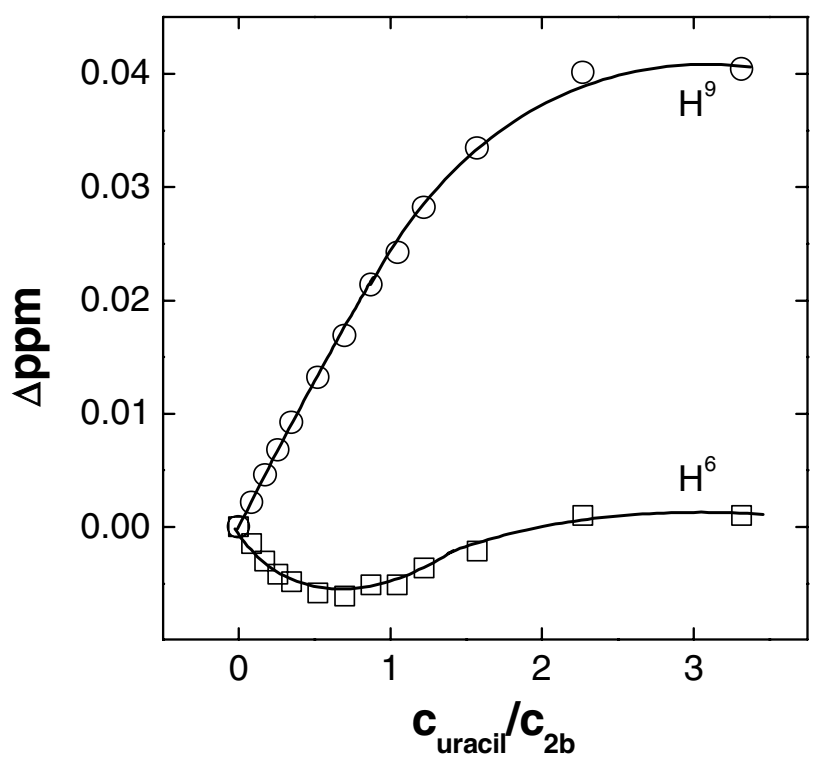


Fig. 4. Relative chemical shifts in ${ }^{1} \mathrm{H}$ NMR spectra of $\mathbf{2} \mathbf{b}$ used for the calculation of binding constants between $\mathbf{2 b}$ and dihydrouracil. Initial concentration $c_{2 \mathrm{~b}}=1.010 .10^{-2} \mathrm{~mol}^{-1} \mathrm{I}^{-1}$.

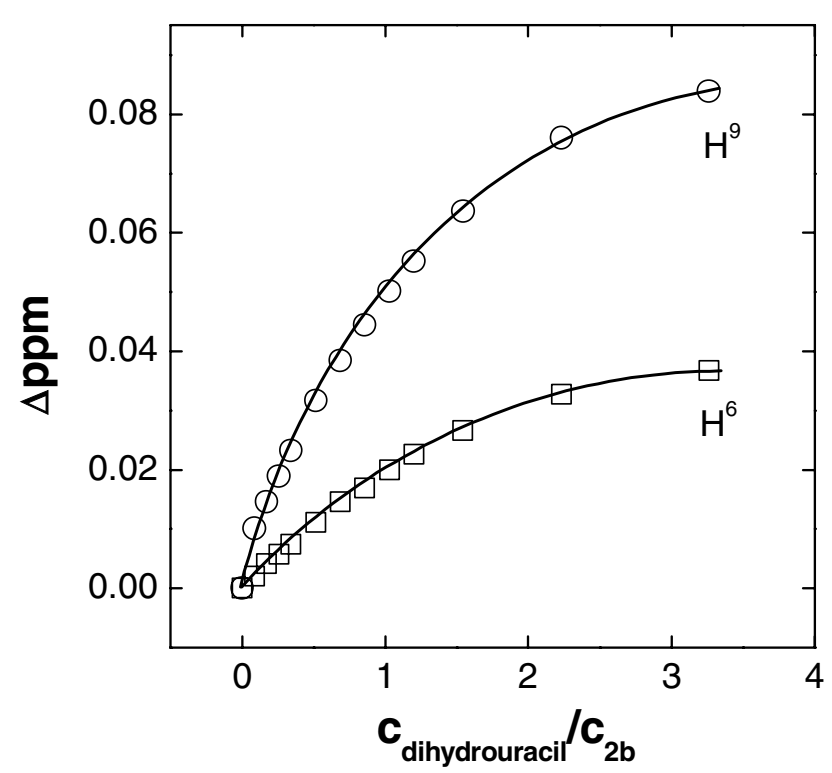

\section{Cycloreversion Assay:}

A mixture of dimer 3a and either the complex $\mathbf{2 a}$ or the flavin derivative $\mathbf{5}$ in acetonitrile or methanol was mixed with an internal standard (acetanilide) in known concentrations. Following Carell's procedure for the flavin reduction, ${ }^{7}$ triethylamine and sodium dithionite were added after degassing by $\mathrm{N}_{2}$. The two samples were irradiated in parallel in methanol experiments or successively in acetonitrile experiments by a Hanovia 450W mercury lamp, equipped with a Pyrex filter. Samples were taken out at regular intervals and analyzed by HPLC (Waters 600 pump and control module, autosampler 717+, PDA 996, Millenium control software) from a gradient acetonitrile-water/TFA $(0.075 \% \mathrm{v} / \mathrm{v}, 70 \%$ to $40 \%$ in $25 \mathrm{~min}$.) on a reverse phase analytical column (Lichrospher C18, $4.6 \times 250 \mathrm{~mm}$ ). The identity of 1a was confirmed by co-injection with an authentic sample of 1a. The assay for the cycloreversion of $\mathbf{3 b}$ by $\mathbf{2} \mathbf{b}$ in water was performed in analogy.

7 Carell, T.; Epple, R.; Gramlich, V. Angew. Chem. Intl. Ed. Engl. 1996, 35, 620. 